\title{
Effect of different geometrical structure of scapula on functional recovery after shoulder arthroscopy operation
}

\author{
Xuchao Shi, Yuanlin Xu, Bo Dai, Weilong Li and Zhennian He*
}

\begin{abstract}
Background: There are no published studies of the influence of geometry of the scapula on the postoperative recovery of rotator cuff injuries. Our aim was to explore the relationship between the critical shoulder angle (CSA), acromion index (Al), glenoid inclination (Gl), and postoperative repair outcomes in shoulder joints after arthroscopic supraspinatus tendon repair.

Methods: Sixty two patients suffering a supraspinatus tear were analyzed retrospectively following failure of conservative treatment and subsequent shoulder arthroscopy in our hospital. Standard anterior and posterior X-rays of the injured shoulder had been performed prior to surgery, with follow ups for at least 2 years (24-43 months). Magnetic resonance imaging (MRI) was performed 2 years after surgery to assess repair of the supraspinatus tendon. Patients were divided into either the intact or re-tear group, according to the MRI results. In addition, assessments using the Constant Shoulder Score (CSS), the American Shoulder and Elbow Surgeon (ASES) Shoulder Assessment Form, the University of California at Los Angeles (UCLA) score and visual analog scale (VAS) score were performed to establish shoulder function at the 2-year evaluation for each patient.

Results: The mean CSA of all patients was $35.79^{\circ} \pm 3.59^{\circ}$, mean Al was $0.72 \pm 0.05$, and mean Gl was $15.87^{\circ} \pm 3.62^{\circ}$. The CSA, Al, and $\mathrm{Gl}$ in the intact group were statistically significantly different than the re-tear group $(p<0.05)$. There was no correlation between the magnitude of the CSA, Al, or Gl and any shoulder function score $(p>0.05)$.

Conclusions: The geometry of the scapula had no significant effect on the recovery of postoperative function of patients with rotator cuff injury, but the value of the CSA, Al, and Gl affected the risk of rotator cuff re-tear.
\end{abstract}

Keywords: Critical shoulder angle, Acromion index, Glenoid inclination, Functional recovery

\section{Background}

Tearing of the rotator cuff is a common injury of the shoulder joint. Its pathogenesis is related to many factors. Many reports have been published that demonstrate that the risk of such injuries and recurrence post-repair are related to age, smoking, initial tear size, and fatty infiltration [1-6]. Recent studies [4-7] have demonstrated that differences in scapula geometry also affect the incidence of rotator cuff tears (RCTs), including the critical shoulder angle (CSA), acromion index (AI), or glenoid inclination (GI).

The AI is obtained by measuring the distance from the glenoid plane to the lateral border of the acromion (GA)

\footnotetext{
* Correspondence: shixuchao0574@163.com

Department of Orthopaedics Surgery of Beilun People's Hospital, No. 1288, Lushan East Road, Ningbo 315800, Zhejiang Province, China
}

divided by the distance from the glenoid plane to the lateral aspect of the humeral head (GH) [2]. The CSA is a combination of AI and GI [7, 8]; it is the angle formed from a line parallel to the glenoid where it crosses a line from the most inferior part of the glenoid and the most lateral edge of the inferior acromion (Fig. 1). Studies have shown that having a CSA $>35^{\circ}$ is associated with RCTs, while a CSA $<30^{\circ}$ is associated with osteoarthritis of the glenohumeral joint [912]. In order to establish the relationship between CSA and RCTs, researchers have previously conducted studies on biomechanics. Gerber et al. [13] demonstrated that load and compressive forces on the supraspinatus tendon increased significantly when the CSA $>38^{\circ}$, compared with CSAs $<38^{\circ}$. In addition, Moor et al. [7] demonstrated that when CSA $>35^{\circ}$, stress on the shoulder joint was

(C) The Author(s). 2019 Open Access This article is distributed under the terms of the Creative Commons Attribution 4.0 International License (http://creativecommons.org/licenses/by/4.0/), which permits unrestricted use, distribution, and 


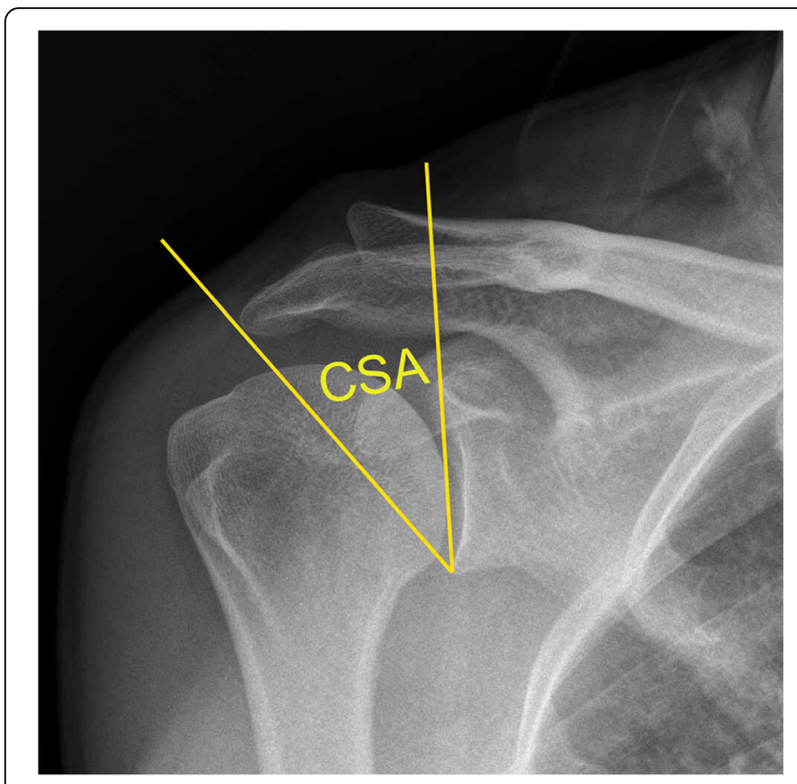

Fig. 1 The critical shoulder angle (CSA) is the angle formed from a line parallel to the glenoid where it crosses a line from the most inferior part of the glenoid and the most lateral edge of the inferior acromion

significantly altered, resulting in an increased risk of humeral translation. Previous studies have demonstrated that the size of the AI facilitates RCTs [14-16]. Although many publications have discussed the impact of various geometries of the scapula on RCTs, no study has convincingly compared the effects of CSA on postoperative repair of a rotator cuff tears (RCT). MRI is a non-invasive tool that can readily observe changes in the integrity of tendons and muscle atrophy after repair of an RCT.

The main purpose of our study was to investigate the effects of different values of CSA, AI, and GI on repair of the supraspinatus after an RCT, with evaluation of the effects of these parameters through the clinical performance of the shoulder joint after surgery.

\section{Methods}

\section{Patient selection}

Patients diagnosed with a simple supraspinatus tendon tear from April 2010 to June 2014, who had failed conservative treatment and had therefore undergone shoulder arthroscopy in our hospital were selected. The study was approved by the local institutional review board. All patients provided written informed consent prior to allocation into the study. Our inclusion criteria for patients were (1) symptomatic patients with a full-thickness supraspinatus tear evaluated through shoulder ultrasound or MRI, for whom conservative treatment had failed and (2) preoperative anterior and posterior shoulder X-rays were performed. Exclusion criteria were (1) multiple tears of the rotator cuff except of the supraspinatus, (2) history of shoulder surgery, and (3) presence of degenerative or neuromuscular diseases of the shoulder joint.

\section{Surgical technique}

All shoulder surgery was performed by a senior surgeon $(\mathrm{HZN})$. All patients underwent general anesthesia and were placed in a beach chair position, which reduced blood pressure, with systolic blood pressure controlled at approximately $95 \mathrm{mmHg}$ intraoperatively. Using a posterior approach with an arthroscope, the anterior joint capsule, the labrum, the rotator cuff, the glenoid, and the cartilage of the humeral head were explored with the biceps muscle tendon as the starting point. An anterior approach assessed the labrum and posterior capsule and explored the subacromial gap. After exploration, capsulotomy was performed to remove any edema or hypertrophic synovial tissue in the glenohumeral joint cavity. During this intervention, free the rotator cuff to prevent the tension from being too high when it was pulled to the position where it needs to be fixed. A standard rotator cuff repair was then performed using a double row technique: 2 threaded suture anchors were placed through the rotator cuff, $45^{\circ}$ to the humerus, with the rotator cuff suspended from the articular surface.

\section{Postoperative rehabilitation}

Shoulder position was immobilized in a neutral position for the 4 weeks following surgery. Gentle passive functional exercise started on the first day after surgery, with internal and external rotation activities or a pendulum activity performed without pain. Active exercise began after 2 weeks, with active flexion to positions higher than the shoulder joint generally starting 4-6 weeks after surgery. At this time, strength training of the shoulder joint was initiated, with full recovery in levels of activity taking 3-6 months.

\section{Patient assessment}

Initial assessment of the patient was performed using a shoulder X-ray. The CSA, AI, and GI were measured by two physicians (SXC, XYL) with the order in which they were measured randomized. The CSA is the angle formed by the intersection of a line connecting the upper and lower edges of the glenoid and a line connecting the outermost point of the shoulder to the lower edge of the glenoid (Fig. 1) [7, 8, 17]. To measure AI, the $\mathrm{GA}$ and $\mathrm{GH}$ were measured separately, with $\mathrm{AI}=$ GA/GH (Fig. 2) [7]. The beta angle is formed by the intersection of a line passing through the floor of the supraspinatus fossa and the line connecting the upper and lower glenoid (Fig. 3) [18]. GI is calculated as $90^{\circ}$ minus the beta angle.

A functional assessment of the shoulder was performed 2 years after surgery, using Constant Shoulder Score 


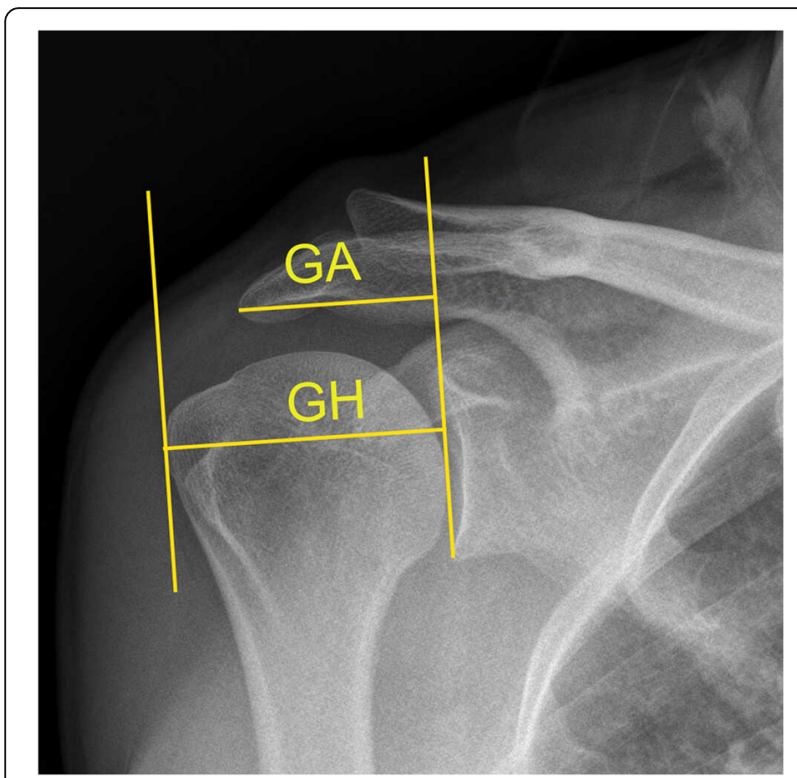

Fig. 2 The acromion index (Al) is obtained by measuring the distance from the glenoid plane to the lateral border of the acromion (GA) divided by the distance from the glenoid plane to the lateral aspect of the humeral head $(\mathrm{GH})$

(CSS), American Shoulder and Elbow Surgeon (ASES), and UCLA scores. The VSA score was used to assess postoperative shoulder pain, using a pain score from 0 to 10 , with 0 indicating no pain at all and 10 indicating the most severe pain the patient could imagine.

At the 2-year follow-up, patients also underwent a shoulder examination using a 1.5-T MRI. Postoperative

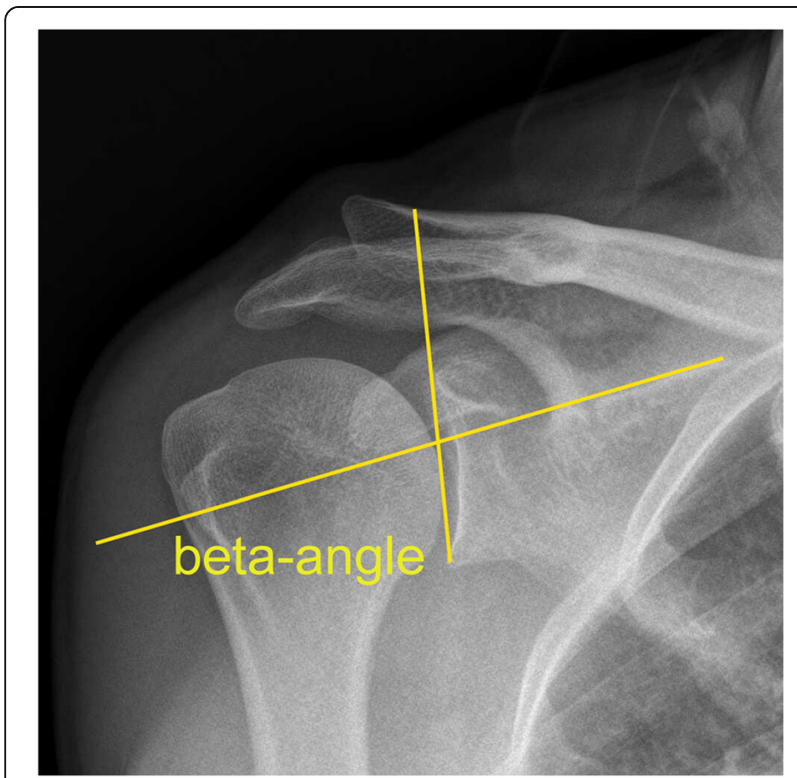

Fig. 3 The glenoid inclination (Gl) is formed by the intersection of a line passing through the floor of the supraspinatus fossa and the line connecting the upper and lower glenoid cuff integrity was classified into 5 categories using oblique coronal, oblique sagittal, and transverse views of T2weighted images: Type I, the repaired rotator cuff appeared to be of a thickness on each image that would have uniform low strength compared to a normal cuff; Type II, thick enough compared with a normal cuff associated with some high-intensity areas; Type III, cuff thickness less than half of the normal cuff, but with no discontinuity, indicating partial thickness tearing; Type IV, only a slight discontinuity in 1 or 2 slices on the slanted coronal and sagittal images, indicating a small full-thickness tear; Vtype, major discontinuities in more than 2 slices observed on the oblique coronal and sagittal images, indicating a medium or large full-thickness tear [19]. Repair integrity was classified into two categories: intact or torn (full thickness re-tear, Sugaya Type IV or V [2]).

\section{Statistical analysis}

Statistical analysis was performed using SPSS 22.0 (IBM Corporation, Armonk, NY, USA). All count data were described in terms of mean and SD and compared using an independent-samples $t$ test. All measurements were compared using a chi-square test. A Pearson correlation was used to assess the relationship between CSA, AI, GI, and shoulder function scores.

Inter- and intra-observer reliability were quantified using the intraclass correlation coefficient (ICC), which ranges from 0 to 1.0, with higher values indicating better reliability. An ICC of 0.0-0.25 indicating little correlation, 0.26-0.49 indicating low correlation, 0.50-0.69 indicating moderate correlation, 0.70-0.89 indicating high correlation, and 0.90-1.00 indicating very high correlation.

\section{Results}

\section{Demographics}

A total of 62 patients were followed up. Mean follow-up time was $34 \pm 4$ months (24-43 months). MRIs revealed that 34 patients had an intact rotator cuff 2 years after surgery, while 28 patients had rotator cuff re-tears. The demographic characteristics of all patients are shown in Table 1. No significant difference existed in the demographic characteristics of the intact group compared with the re-tear group $(p>0.05)$.

\section{Radiographic measurement}

The CSA, AI, and GI were measured for each patient. Mean CSA was $35.79^{\circ} \pm 3.59^{\circ}$ for all patients, mean AI was $0.72 \pm 0.05$, and mean GI was $15.87^{\circ} \pm 3.62^{\circ}$. It can be seen from Table 1 that both the CSA and AI in the re-tear group were significantly larger than in the intact group $(p<0.05)$. However, in the intact group, the GI was significantly larger than in the re-tear group $(p<0.05)$. 
Table 1 Different characteristics between intact group and retear group

\begin{tabular}{llll}
\hline & Intact group $(n=34)$ & Re-tear group $(n=28)$ & $p$ \\
\hline Age & $52.76 \pm 6.38$ & $51.14 \pm 6.40$ & .324 \\
BMI & $22.38 \pm 2.75$ & $21.86 \pm 2.79$ & .460 \\
Sex & & & \\
$\quad$ Male & 16 & 17 & .284 \\
$\quad$ Female & 18 & 11 & \\
Side & & & .895 \\
$\quad$ Left & 9 & 7 & \\
Right & 25 & 21 & .008 \\
CSA & $34.74 \pm 3.25$ & $37.07 \pm 3.40$ & .006 \\
Al & $0.71 \pm 0.04$ & $0.74 \pm 0.05$ & .021 \\
Gl & $16.82 \pm 3.51$ & $14.71 \pm 3.46$ & .085 \\
CSS & $73.35 \pm 6.15$ & $76.04 \pm 5.83$ & .911 \\
ASES & $87.68 \pm 4.37$ & $87.53 \pm 5.47$ & .200 \\
UCLA & $29.65 \pm 2.77$ & $30.57 \pm 2.82$ & .311 \\
VAS & $1.32 \pm 1.07$ & $1.64 \pm 1.39$ & \\
\hline
\end{tabular}

$B M I$ body mass index, CSA critical shoulder angle, $A$ l acromial index, Gl glenoid inclination, CSS Constant Shoulder Score, ASES American Shoulder and Elbow Surgeon, UCLA University of California at Los Angeles, VAS visual analog scale

\section{Result score}

Functional shoulder assessments were also evaluated on patients 2 years after surgery, including CCA, ASES, UCLA, and VAS scores. As can be seen from Table 2, no significant correlation existed between the values of CSA, AI, or GI with any shoulder joint evaluation indicator $(p>0.05)$. In addition, in Table 1 , there was no difference in shoulder function in the intact group compared with the re-tear group $(p>0.05)$.

\section{Results of reliability analysis}

Measurement of inter-observer reliability of radiographic images resulted in an ICC value of 0.945 for CSA, 0.938 for AI, and 0.908 for GI (Table 3).

Table 2 Relationship between the result of radiographic measurement and functional scores of shoulder joint 2 years after operation

\begin{tabular}{|c|c|c|c|c|c|c|}
\hline & \multicolumn{2}{|l|}{ CSA } & \multicolumn{2}{|l|}{$\underline{\mathrm{Al}}$} & \multicolumn{2}{|l|}{ Gl } \\
\hline & $r$ & $p$ & $r$ & $p$ & $r$ & $p$ \\
\hline CSS & 0.23 & .063 & 0.09 & .475 & 0.22 & .073 \\
\hline ASES & -0.02 & .887 & 0.19 & .140 & -0.08 & .536 \\
\hline UCLA & -0.17 & .168 & 0.05 & .707 & -0.11 & .384 \\
\hline VAS & -0.15 & .258 & 0.15 & .230 & 0.19 & .136 \\
\hline
\end{tabular}

CSA critical shoulder angle, Al acromial index, Gl glenoid inclination, CSS Constant Shoulder Score, ASES American Shoulder and Elbow Surgeon, UCLA University of California at Los Angeles, VAS visual analog scale
Table 3 ICCs for inter-observer and intra-observer reliability

\begin{tabular}{llll}
\hline & CSA & Al & Gl \\
\hline ICC & .945 & .938 & .908 \\
\hline
\end{tabular}

CSA critical shoulder angle, Al acromial index, Gl glenoid inclination, ICC Intraclass correlation coefficient

\section{Discussion}

Prior to this study, many reports have been published discussing the effects of differences in geometry of the scapula such as the CSA, AI, or GI on RCTs and shoulder osteoarthritis [20-23]. In these studies, the magnitude of the measurements was shown to greatly affect the incidence of shoulder joint disease. However, the long-term effects of the value of the CSA on the healing of RCT repairs have not been studied.

The mean CSA of all patients was $35.79^{\circ} \pm 3.59^{\circ}$ in our study, similar to that measured in previously published literature [24, 25]. Garcia et al. [26] used ultrasound to establish the effect of high CSA values on postoperative repair of rotator cuffs. The results demonstrated that large angles significantly increased the risk of tendon tear recurrence after repair of the rotator cuff. In our study, patients with CSA $>38^{\circ}$ had a threefold higher risk of rotator cuff re-tear than patients with a $\operatorname{CSA}<38^{\circ}$. This indicates that a high CSA increases the risk of re-tear after a single supraspinatus tear. A biomechanical study by Gerber et al. [27] demonstrated that high CSA can cause an overload of the supraspinatus tendon during active abduction of the shoulder joint, which is consistent with our study. Because of the effect of a high CSA on rotator cuff injury, researchers have conducted cadaveric studies that reduced the lateral extension of the CSA by performing a lateral acromioplasty to reduce lateral extension of the acromion $[28,29]$. In our study, the acromion did not undergo lateral resection, so patients with a high CSA were still subjected to a high supraspinatus load after surgery. This maybe a good explanation for why the re-tear rate of the rotator cuff in the high CSA patients in our study was lower than that in the low CSA patients.

Many studies have reported the relationship between $\mathrm{AI}$ and degenerative cuff tears, with a large AI increasing the risk of RCT [16, 30, 31]. In our study, mean AI was $0.72 \pm 0.05$. There was a significant difference between the $\mathrm{AI}$ and re-tear groups, indicating that the value of $\mathrm{AI}$ affected the recovery from RCT repairs, similar to the results of Zumstein et al. [32]. Ames et al. [14] followed up for 2 years after RCT surgery, finding that high AI reduced patient satisfaction. These results were similar to Lee et al. [33]. In our study, there was no significant correlation between AI and postoperative function.

GI has been shown to be a risk factor in supraspinatus tears [34-36]. The present study showed that mean GI was significantly different in the intact and re-tear groups $(p<0.05)$. For a $\mathrm{GI}>14^{\circ}$, the risk of patient rotator cuff 
re-tear was five times higher. Gerber et al. [13] reported similar findings, in that high GI significantly increased the risk of re-tear after rotator cuff repair.

In our 2-year follow-up, patients completed a questionnaire and underwent shoulder function tests (CCA, ASES, UCLA, VAS). Our results indicated that there was no significant correlation in patient's postoperative function and CSA, AI, or GI. Lee et al. [33] also compared the relationship between CSA, AI, and postoperative shoulder function, with similar results to the present study, with no significant relationship between any parameter. In addition, our results showed that there was no significant relationship between the integrity of the rotator cuff and shoulder joint function following surgery. Patient rotator cuff re-tear does not necessarily lead to functional deterioration. Millett et al. [37] demonstrated that patients with RCTs are not necessarily symptomatic, sometimes taking more than 2 years for symptoms to develop. Further follow-up studies are required to ascertain whether rotator cuff re-tears further affect shoulder function.

There are some limitations in our research. Firstly, the sample size was small, so the results may be biased. Secondly, even though the imaging measurements were reliable, as this was a retrospective study, the angle of the shoulder joint in the X-rays may not have been correctly positioned. Finally, MRI and shoulder function were only tested 2 years after surgery, and no short-term tests were performed, so the dynamic relationship between shoulder function and rotator cuff repair could not be fully evaluated.

\section{Conclusions}

This study demonstrated that although higher CSA increased the risk of rotator cuff re-tear, there was no significant effect on shoulder function after rotator cuff repair. Similarly, the magnitude of AI and GI did not affect shoulder joint function after rotator cuff repairs. It can be seen that the specific geometry of the scapula affects the repair of the rotator cuff after shoulder surgery, but not the recovery of shoulder function.

\section{Abbreviations}

Al: Acromial index; ASES: American Shoulder and Elbow Surgeon; CSA: Critical shoulder angle; CSS: Constant Shoulder Score; Gl: Glenoid inclination; ICC: Intraclass correlation coefficient; MRI: Magnetic resonance imaging; RCT: Rotator cuff tears; UCLA: University of California at Los Angeles; VAS: Visual analog scale

\section{Acknowledgements}

The authors would like to thank Dr. Lun Zhang for his help with this study. This research was performed at Department of Orthopaedics Surgery of Beilun People's Hospital, No. 1288, Lushan East Road, Ningbo, Zhejiang Province, China.

\section{Authors' contributions}

$X C S, Z N H$, and $W L L$ designed the study, $B D, X C S$, and ZNH collected the data. WLL, XCS, and BD analyzed the data. XCS, YLX, and ZNH interpreted the data. ZNH, YLX, and XCS composed the article. All authors read and approved the final manuscript.

Funding

No funding was obtained for this study.

Availability of data and materials

Not applicable

Ethics approval and consent to participate

The study was approved by the Ethical Board Review of the Beilun People's Hospital (Ningbo, Zhejiang, China), participant consent was written, and the reference number was ChiCTR1800019089 and was performed in accordance with the ethical standards of the Declaration of Helsinki of 1964.

\section{Consent for publication}

Not applicable

\section{Competing interests}

The authors declare that they have no competing interests.

Received: 20 November 2018 Accepted: 5 September 2019

Published online: 14 September 2019

\section{References}

1. Flurin PH, Hardy P, Abadie P, et al. Arthroscopic repair of the rotator cuff: prospective study of tendon healing after 70 years of age in 145 patients. Orthop Traumatol Surg Res. 2013;99(8 Suppl):S379-84.

2. Lee YS, Jeong JY, Park CD, et al. Evaluation of the risk factors for a rotator cuff retear after repair surgery. Am J Sports Med. 2017;45(8):1755-61.

3. Tashjian RZ, Hollins AM, Kim HM, et al. Factors affecting healing rates after arthroscopic double-row rotator cuff repair. Am J Sports Med. 2010;38(12):2435-42.

4. Minagawa $\mathrm{H}$, Yamamoto $\mathrm{N}, \mathrm{Abe} \mathrm{H}$, et al. Prevalence of symptomatic and asymptomatic rotator cuff tears in the general population: from mass-screening in one village. J Orthop. 2013;10(1):8-12.

5. Bishop JY, Santiago-Torres JE, Rimmke N, et al. Smoking predisposes to rotator cuff pathology and shoulder dysfunction: a systematic review. Arthroscopy. 2015;31(8):1598-605.

6. Lazarides AL, Alentorn-Geli E, Choi JHJ, et al. Rotator cuff tears in young patients: a different disease than rotator cuff tears in elderly patients. J Shoulder Elb Surg. 2015;24(11):1834-43.

7. Moor BK, Kuster R, Osterhoff G, et al. Inclination-dependent changes of the critical shoulder angle significantly influence superior glenohumeral joint stability. Clin Biomech. 2016:32:268-73.

8. Blonna D, Giani A, Bellato $E$, et al. Predominance of the critical shoulder angle in the pathogenesis of degenerative diseases of the shoulder. J Shoulder Elb Surg. 2016;25(8):1328-36.

9. Peltz CD, Divine G, Drake A, et al. Associations between in-vivo glenohumeral joint motion and morphology. J Biomech. 2015;48(12):3252-7.

10. Kim JR, Ryu KJ, Hong IT, et al. Can a high acromion index predict rotator cuff tears? Int Orthop. 2012;36(5):1019-24.

11. Pandey V, Vijayan D, Tapashetti S, et al. Does scapular morphology affect the integrity of the rotator cuff? J Shoulder Elb Surg. 2016;25(3):413-21.

12. Spiegl UJ, Horan MP, Smith SW, et al. The critical shoulder angle is associated with rotator cuff tears and shoulder osteoarthritis and is better assessed with radiographs over MRI. Knee Surg Sports Traumatol Arthrosc. 2016;24(7):2244-51.

13. Gerber C, Snedeker JG, Baumgartner D, et al. Supraspinatus tendon load during abduction is dependent on the size of the critical shoulder angle: a biomechanical analysis. J Orthop Res. 2014;32(7):952-7.

14. Ames JB, Horan MP, Van der Meijden OA, et al. Association between acromial index and outcomes following arthroscopic repair of full-thickness rotator cuff tears. J Bone Joint Surg Am. 2012;94(20):1862-9.

15. Kircher J, Morhard M, Gavriilidis I, et al. Is there an association between a low acromion index and osteoarthritis of the shoulder? Int Orthop. 2010;34(7):1005-10.

16. Miyazaki AN, Itoi $\mathrm{E}$, Sano $\mathrm{H}$, et al. Comparison between the acromion index and rotator cuff tears in the Brazilian and Japanese populations. J Shoulder Elb Surg. 2011;20(7):1082-6. 
17. Bhatia DN, Debeer JF, du Toit DF. Association of a large lateral extension of the acromion with rotator cuff tears. J Bone Joint Surg Am. 2006;88(8):1889 author reply 1889-90.

18. Maurer A, Fucentese SF, Pfirrmann CW, et al. Assessment of glenoid inclination on routine clinical radiographs and computed tomography examinations of the shoulder. J Shoulder Elb Surg. 2012;21(8):1096-103.

19. Sugaya $\mathrm{H}$, Maeda K, Matsuki K, et al. Functional and structural outcome after arthroscopic full-thickness rotator cuff repair: single-row versus dual-row fixation. Arthroscopy. 2005;21(11):1307-16.

20. Aydin N, Kocaoglu B, Guven O. Single-row versus double-row arthroscopic rotator cuff repair in small- to medium-sized tears. J Shoulder Elb Surg. 2010;19(5):722-5.

21. Khazzam M, Kuhn JE, Mulligan E, et al. Magnetic resonance imaging identification of rotator cuff retears after repair: interobserver and intraobserver agreement. Am J Sports Med. 2012;40(8):1722-7.

22. Lapner PL, Sabri E, Rakhra K, et al. A multicenter randomized controlled trial comparing single-row with double-row fixation in arthroscopic rotator cuff repair. J Bone Joint Surg Am. 2012;94(14):1249-57.

23. Zanetti M, Jost B, Hodler J, et al. MR imaging after rotator cuff repair: fullthickness defects and bursitis-like subacromial abnormalities in asymptomatic subjects. Skelet Radiol. 2000;29(6):314-9.

24. Moor BK, Bouaicha S, Rothenfluh DA, et al. Is there an association between the individual anatomy of the scapula and the development of rotator cuff tears or osteoarthritis of the glenohumeral joint?: a radiological study of the critical shoulder angle. Bone Joint J. 2013;95-B(7):935-41.

25. Moor BK, Wieser K, Slankamenac K, et al. Relationship of individual scapular anatomy and degenerative rotator cuff tears. J Shoulder Elb Surg. 2014;23(4):536-41.

26. Garcia GH, Liu JN, Degen RM, et al. Higher critical shoulder angle increases the risk of retear after rotator cuff repair. J Shoulder Elb Surg. 2017;26(2):241-5.

27. Gerber C, Rahm SA, Catanzaro S, et al. Latissimus dorsi tendon transfer for treatment of irreparable posterosuperior rotator cuff tears: long-term results at a minimum follow-up of ten years. J Bone Joint Surg Am. 2013:95(21):1920-6.

28. Altintas B, Kaab M, Greiner S, et al. Arthroscopic lateral acromion resection (ALAR) optimizes rotator cuff tear relevant scapula parameters. Arch Orthop Trauma Surg. 2016;136(6):799-804.

29. Katthagen JC, Marchetti DC, Tahal DS, et al. The effects of arthroscopic lateral acromioplasty on the critical shoulder angle and the anterolateral deltoid origin: an anatomic cadaveric study. Arthroscopy. 2016;32(4):569-75.

30. Nyffeler RW, Werner CM, Sukthankar A, et al. Association of a large lateral extension of the acromion with rotator cuff tears. J Bone Joint Surg Am. 2006;88(4):800-5.

31. Balke M, Schmidt C, Dedy N, et al. Correlation of acromial morphology with impingement syndrome and rotator cuff tears. Acta Orthop. 2013;84(2):178-83.

32. Zumstein MA, Jost B, Hempel J, et al. The clinical and structural long-term results of open repair of massive tears of the rotator cuff. J Bone Joint Surg Am. 2008;90(11):2423-31.

33. Lee M, Chen JY, Liow MHL, et al. Critical shoulder angle and acromial index do not influence 24-month functional outcome after arthroscopic rotator cuff repair. Am J Sports Med. 2017;45(13):2989-94.

34. Daggett $M$, Werner $B$, Collin $P$, et al. Correlation between glenoid inclination and critical shoulder angle: a radiographic and computed tomography study. J Shoulder Elb Surg. 2015;24(12):1948-53.

35. Wong AS, Gallo L, Kuhn JE, et al. The effect of glenoid inclination on superior humeral head migration. J Shoulder Elb Surg. 2003;12(4):360-4.

36. Bishop JL, Kline SK, Aalderink KJ, et al. Glenoid inclination: in vivo measures in rotator cuff tear patients and associations with superior glenohumeral joint translation. J Shoulder Elb Surg. 2009;18(2):231-6.

37. Millett PJ, Warth RJ, Dornan GJ, et al. Clinical and structural outcomes after arthroscopic single-row versus double-row rotator cuff repair: a systematic review and meta-analysis of level I randomized clinical trials. J Shoulder Elb Surg. 2014;23(4):586-97.

\section{Publisher's Note}

Springer Nature remains neutral with regard to jurisdictional claims in published maps and institutional affiliations.

Ready to submit your research? Choose BMC and benefit from:

- fast, convenient online submission

- thorough peer review by experienced researchers in your field

- rapid publication on acceptance

- support for research data, including large and complex data types

- gold Open Access which fosters wider collaboration and increased citations

- maximum visibility for your research: over $100 \mathrm{M}$ website views per year

At BMC, research is always in progress.

Learn more biomedcentral.com/submissions 